\title{
NEURILEMOMA DE LÍNGUA: CONSIDERAÇÕES GERAIS E RELATO DE CASO CLÍNICO
}

NEURILEMOMA OF THE TONGUE: GENERAL CONSIDERATIONS AND REPORT OF A CASE

\author{
Fernando Paganeli Machado Giglio * \\ Luís Antônio de Assis Taveira \\ Luiz Eduardo Montenegro Chinellato
}

\section{RESUMO}

O neurilemoma é um tumor benigno raro de origem neuroectodérmica que pode acometer a boca, onde tem notada predileçâo pela língua. Devido às suas características clínicas e de desenvolvimento, é uma condição que pode passar desapercebida pelo paciente durante anos até atingir maiores tamanhos e confundir o próprio clínico por mimetizar outras lesôes. O objetivo do presente artigo é salientar os principais aspectos inerentes ao diagnóstico e tratamento dos neurilemomas, além de relembrar suas peculiaridades clínicas e microscópicas, por meio da ilustração de um caso clínico.

DESCRITORES: Neurilemoma $\bullet$ Doenças da língua

\section{ABSTRACT}

Neurilemoma is a rare benign tumor of neural origin that can affect the oral cavity, where it has prediction for the tongue. Due to its clinical and development characteristics, it's a condition that might to be unperceivable by the patient during many years until to reach larger sizes and to confuse the own dentist for disguise of other lesions. The aim of the present paper is to point out the most important inherent aspects to the diagnosis and treatment of the neurilemomas, besides remember its clinical and microscopic features, through the illustration of a clinical case.

DESCRIPTORS: Neurilemmoma $\bullet$ Tongue diseases

* Mestre e Doutor em Estomatologia pela Faculdade de odontologia de Bauru (FOB-USP)

** Professor do Departamento de Estomatologia (Patologia) da Faculdade de odontologia de Bauru (FOB-USP)

*** Professor do Departamento de Estomatologia da Faculdade de odontologia de Bauru (FOB-USP) 


\section{INTRODUÇÃO E REVISTA DA LITERATURA}

O neurilemoma, antigamente conhecido como schwannoma, é um tumor benigno de natureza neuroectodérmica, oriundo da proliferaçáo das células de Schwann que envolvem os nervos periféricos (Regizzi e Sciubba ${ }^{14}$ 2000). Essa lesão foi primeiramente descrita por Verocay em 1910, com a denominação de neurinoma; enquanto que o termo neurilemoma foi proposto por Stout em 1935 (Nary Filho et al. ${ }^{11}$ 2002).

Apresenta-se na maioria das vezes como massa submucosa séssil, encapsulada, solitária, macia à palpação e assintomática, em indivíduos de qualquer idade (Baranoic et al. ${ }^{2}$ 2006), com uma aparente predileção pela segunda e terceira décadas - $67 \%$ dos casos (Krolls et al. ${ }^{8}$ 1994). Tem desenvolvimento lento, podendo apresentar surtos de crescimento, possivelmente por hemorragias em seu interior; e, à medida que a lesão cresce, o nervo afetado é deslocado, não ficando envolvido no tumor (Regizzi e Sciubba ${ }^{14}$ 2000); o mesmo ocorre com as mucosas, que são levadas consigo intactas, sendo que as ulceraçôes, quando presentes, são provocadas por traumatismos mecânicos. O tamanho é bastante variável, com boa parte dos casos medindo cerca de 2 centímetros de diâmetro (Hatzioti e Asprides 5 1967).

Entre 20 e 58\% dos neurilemomas de cabeça e pescoço são de origem intrabucal (Amir et al. ${ }^{1}$ 2002); sendo a língua a área mais afetada na boca (Nary Filho et al. ${ }^{11}$ 2002). Não há dados disponíveis na literatura quanto à sua incidência na população.

A duração da lesão antes do tratamento, segundo a literatura, varia de 5 meses a 49 anos, com $45 \%$ dos casos presentes por pelo menos 1 ano, sem que haja maior prevalência evidente em nenhum dos gêneros ou raças (Pfeifle et al. ${ }^{12}$ 2001).

Trata-se de uma situação quase que invariavelmente benigna, apresentando índices extremamente baixos de transformação maligna (Martins et al. ${ }^{9}$ 2007).

Existe também uma variante intraóssea, caracterizada por uma lesão radiolúcida unilocular, que pode causar dor ou parestesia na regiáo afetada, sendo esse tipo bastante raro. As regióes de corpo e ramo da mandíbula são as mais acometidas nesses casos; Chi et al. ${ }^{3}$ (2003) relataram uma prevalência menor que $1 \%$ para o neurilemoma intraósseo dentre os tumores ósseos benignos.

Suas peculiaridades microscópicas são quase patognômonicas, existindo dois padrôes. No primeiro, denominado Antoni A, destacam-se células fusiformes dis- postas em espirais ou ondas, formando uma paliçada; essas células são frequentemente cercadas por uma zona acelular chamada de corpo ou corpúsculo de Verocay, formada por prolongamentos citoplasmáticos interdigitados, membrana basal reduplicada e um pequeno número de fibras colágenas. O segundo padrão, Antoni B, é constituído por células fusiformes distribuídas ao acaso em uma ligeira matriz fibrilar com ausência dos corpos de Verocay. É muito comum ambos os padróes estarem no interior da mesma lesão (Kawakami et al. ${ }^{6}$ 2004). Quanto às características imuno-histoquímicas, o citoplasma das células fusiformes dos neurilemomas apresentam grande positividade para as proteínas S-100,

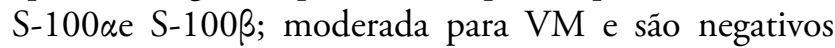
para DM, AT, MS, SMA, GFAP e FVIII; sendo essas informaçóes importantes para o diagnóstico diferencial para a variante maligna e para os leiomiossarcomas (Koizumi et al. ${ }^{7}$ 2002).

\section{PROPOSIÇÃO}

A proposta do presente artigo é salientar os principais aspectos inerentes ao diagnóstico e tratamento dos neurilemomas, relembrando peculiaridades clínicas e microscópicas, por meio da ilustração de um caso clínico.

\section{CASO CLÍNICO}

Paciente R.V.B., 23 anos, gênero masculino, leucoderma, procedente de zona urbana, procurou a clínica de Estomatologia da Faculdade de Odontologia de Bauru da Universidade de Sáo Paulo por orientação de seu ortodontista, que num exame de proservação notou a presença de uma tumefaçáo no dorso da língua. O paciente

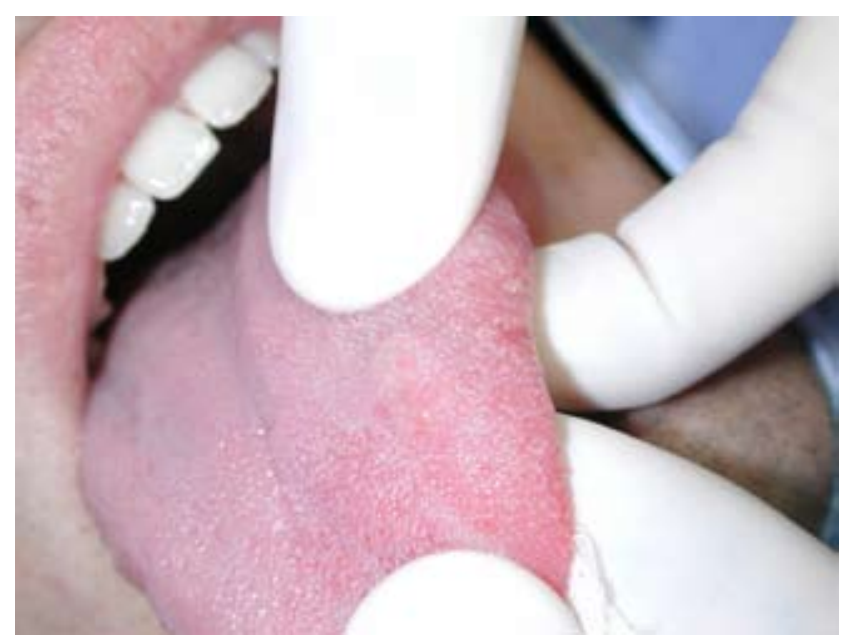

Figura 1 - Aspecto frontal da lesão 
Giglio FPM, Taveira LAA, Chinellato LEM. Neurilemoma de língua: considerações gerais e relato de caso clínico. Revista de Odontologia da Universidade Cidade de São Paulo 2009 mai-ago; 21(2): 179-84

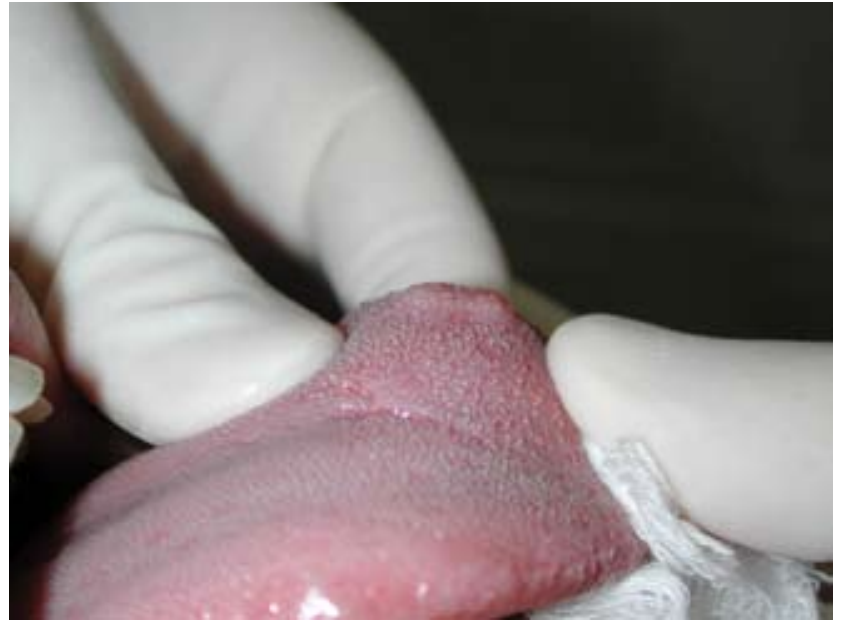

Figura 2 - Aspecto lateral da lesão

mencionou ter ciência do "caroço", com surgimento sem causa definida um ano e meio atrás, sem histórico de dor, sangramento ou drenagem de pus. Os exames geral, locoregional, e sua história médica não apresentaram dados dignos de nota. No exame clínico intrabucal, o único destaque foi um nódulo no dorso lingual, lado esquerdo, medindo cerca de $10 \times 15 \mathrm{~mm}$, sem alteração de cor e superfície ligeiramente lobulada; à palpação, notou-se um aspecto superficial, sendo a lesão fixa, endurecida e de base séssil (Figuras 1 e 2).

Diante de tais características, optou-se pela biópsia excisional, que foi realizada após anestesia infiltrativa à distância, com uma lâmina de bisturi no 15 . A lesão foi removida com margens de aproximadamente $2 \mathrm{~mm}$ e com profundidade suficiente para alcançar o plano mus-

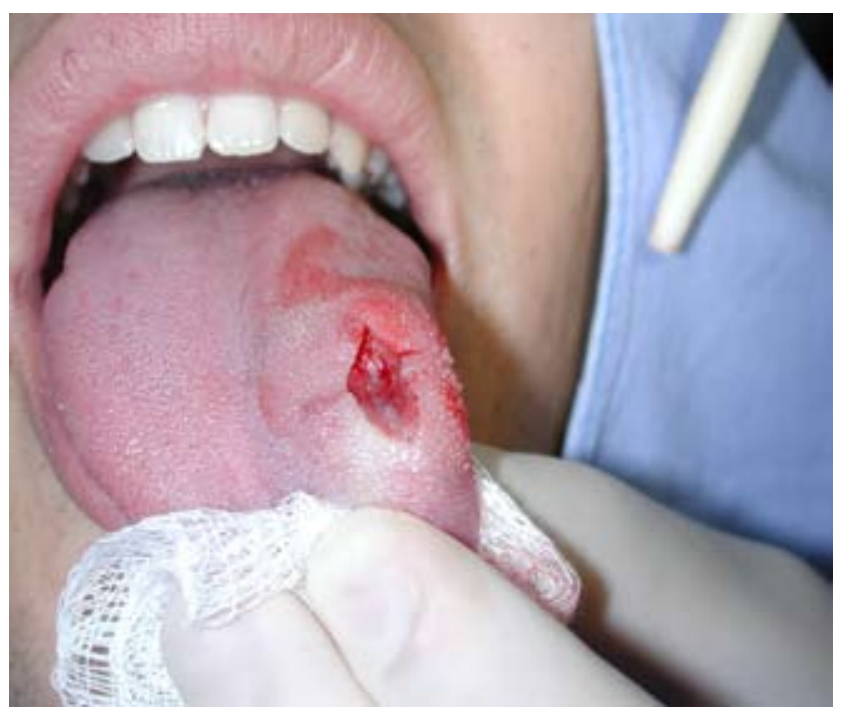

Figura 3 - Leito cirúrgico após a excisão da lesão

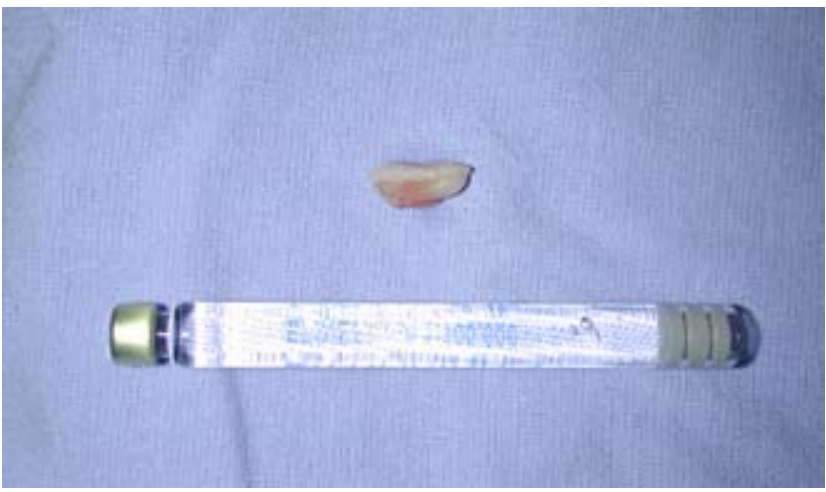

Figura 4 - Peça removida

cular (Figura 3). A síntese do leito cirúrgico foi realizada através de pontos simples com fio de seda 4-0. A peça foi imediatamente imersa em soluçáo de formol a $10 \%$ e enviada à Disciplina de Patologia da FOB-USP para o exame anátomo-patológico, com o diagnóstico presuntivo de lipoma ou linfangioma. $\mathrm{O}$ paciente recebeu as orientaçóes pós-operatórias de rotina e a prescrição de um analgésico, se necessário.

No trans-operatório, pôde-se observar lesão de coloração amarelada, encapsulada, facilmente destacável dos tecidos adjacentes (Figura 4).

A remoção da sutura foi programada para 1 semana após a cirurgia, no entanto, o paciente relatou que os 3 pontos caíram entre o $4^{\circ}$ e o $6^{\circ}$ dias pós-operatórios; a área apresentava-se em processo de reparo e não houve queixas de desconforto. No retorno de 30 dias a região

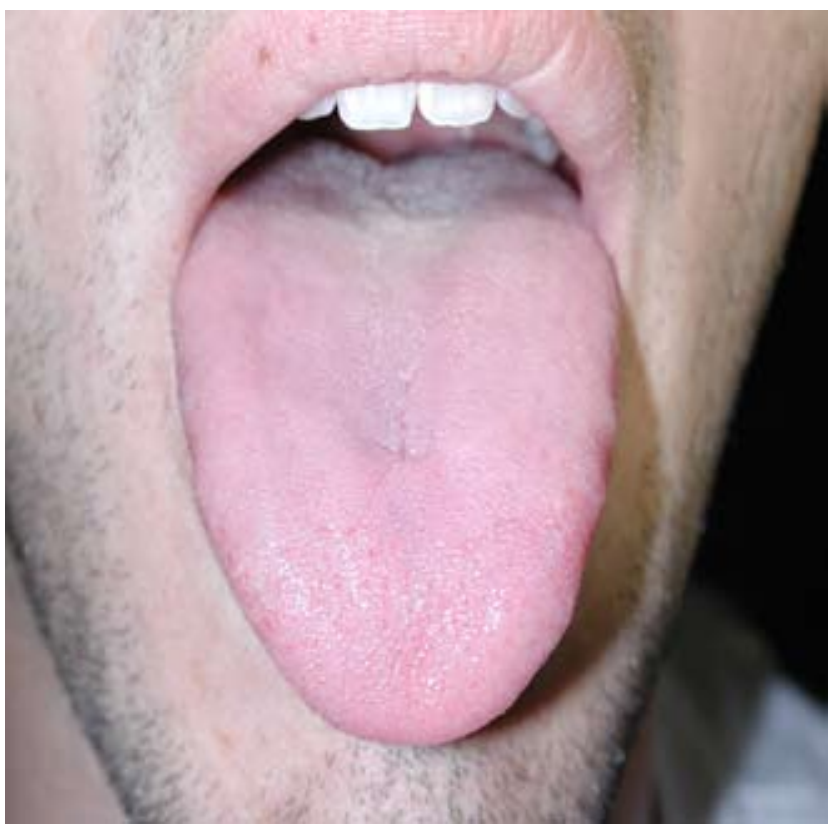

Figura 5 - Completa cicatrização após 30 dias 
Giglio FPM, Taveira LAA, Chinellato LEM. Neurilemoma de língua: considerações gerais e relato de caso clínico. Revista de Odontologia da Universidade Cidade de São Paulo 2009 mai-ago; 21(2): 179-84

estava completamente cicatrizada (Figura 5). É válido ressaltar que o tratamento foi realizado e as fotografias obtidas após a assinatura do termo de consentimento por parte do paciente.

Os cortes microscópicos revelaram proliferação de células fusiformes bem encapsulada e circunscrita, caracterizada por formação de corpúsculos de Verocay. A cápsula fibrosa mostrou-se bem constituída e contínua. Sobrejacente, a mucosa bucal encontrava-se discretamente atrófica, com a lesão localizando-se no plano muscular esquelético e submucoso (Figuras 6 e 7). As características microscópicas levaram ao diagnóstico final de neurilemoma.

\section{DISCUSSÃO}

As células de Schwann formam uma fina barreira, bainha de mielina, ao redor de algumas fibras nervosas periféricas, mielínicas, funcionando como um isolante elétrico, potencializando a condução nervosa, sendo suscetíveis à proliferação tumoral, embora a etiologia ainda permaneça obscura (Pfeifle et al. ${ }^{12}$ 2001).

Sobre a sua prevalência, segundo Pfeifle et al. ${ }^{12}$ (2001), 25\% de todos os neurilemomas estão localizados

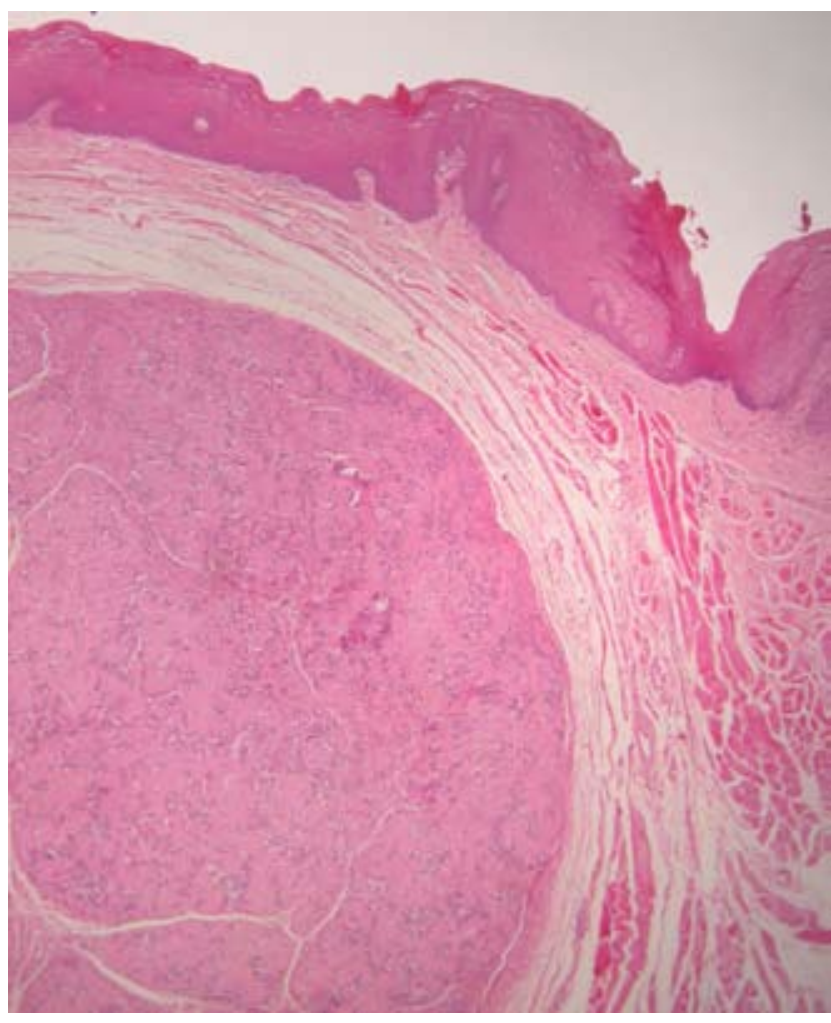

Figuras 6 - Epitélio da mucosa bucal com parte do tumor (notar cápsula fibrosa bem definida)

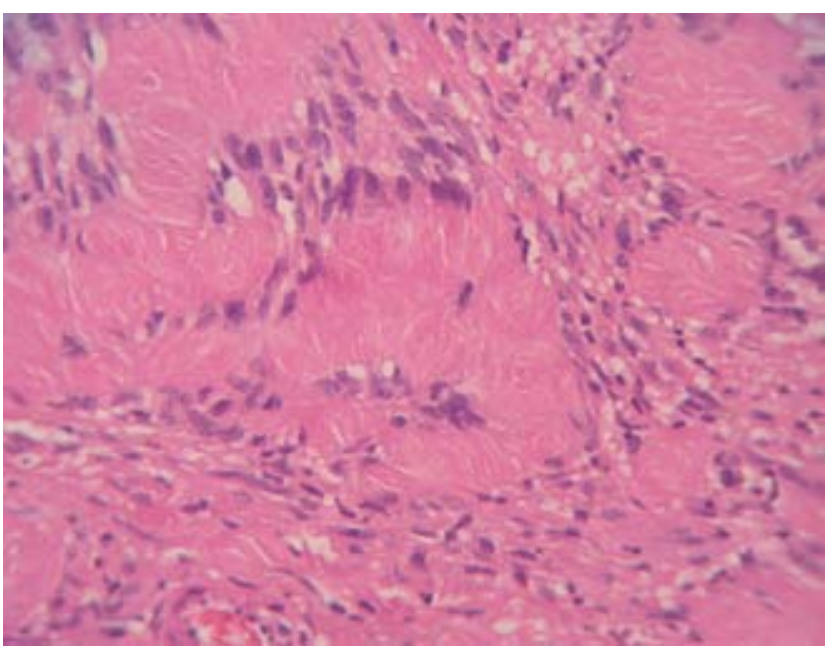

Figura 7 - Na massa tumoral notam-se células fusiformes dispostas em paliçada indicando os corpúsculos de Verocay, juntamente com as áreas hialinas caracteristicas da lesão

na regiáo de cabeça e pescoço, sendo que nessa área cerca de 35\% envolvem a boca e regióes peribucais. A língua é, sem dúvida, a localização intra-bucal mais comum. $\mathrm{Na}$ amostra de Hatziotis e Asprides ${ }^{5}$ (1967), a porcentagem foi de $53 \%$. Outros locais podem ser afetados como palato, mucosa jugal, lábios e rebordos alveolares.

Existem relatos de outras duas formas microscópicas de neurilemoma, além dos padrões Antoni A e B; são os antigos e os celulares. Os antigos parecem tratar-se de neurilemomas de longa duração, que apresentam sinais de degeneração; embora se apresentem clinicamente idênticos aos demais neurilemomas, possuem características anátomo-patológicas distintas, como presença de áreas difusas de hipocelularidade, áreas fibróticas, células inflamatórias, acúmulos de material hialino, degeneração gordurosa, pontos hemorrágicos, além de certas atipias celulares. As características histológicas típicas estão ausentes ou presentes apenas em poucas áreas isoladas (Regizzi e Sciubba ${ }^{14}$ 2000).

Os neurilemomas celulares, recentemente reconhecidos, são muito raros, e, de acordo com Koizumi et al. ${ }^{7}$ (2002), são caracterizados por alta celularidade e ausência de corpos de Verocay, parecendo ocuparem uma posição intermediária entre os neurilemomas benignos e malignos. Frequentemente apresentam características microscópicas de malignidade, tais como hipercromatismo nuclear, pleomorfismo, aumento do número de mitoses, áreas necróticas, além de provocarem erosóes ósseas, dependendo de sua localização. 
Giglio FPM, Taveira LAA, Chinellato LEM. Neurilemoma de língua: considerações gerais e relato de caso clínico. Revista de Odontologia da Universidade Cidade de São Paulo 2009 mai-ago; 21(2): 179-84

No diagnóstico diferencial devem ser considerados os tumores de glândulas salivares, mucoceles, lipomas, neurofibromas, leiomiomas, linfonodos enfartados e fibromas traumáticos. Poucos neurilemomas são diagnosticados clinicamente, pelo fato de essa lesão mimetizar outras condições. É necessário mencionar para o diagnóstico diferencial que apenas 4\% dos neurofibromas são encapsulados, enquanto que $100 \%$ dos neurilemomas o são (Marzola et al. ${ }^{10}$ 1988). Essa discussão sobre a importância da diferenciaçáo entre neurilemomas e neurofibromas é devida ao fato de os neurofibromas possuírem um maior potencial de transformação maligna (Feltrin ${ }^{4}$ 1978). Para as lesóes de tecidos moles, exames como a tomografia computadorizada com janela para tecidos moles, ressonância magnética e ultrassonografia podem contribuir ao diagnóstico (Yamazaki et al. ${ }^{15}$ 2004).

O tratamento de eleição é a excisão cirúrgica, inclusive da mucosa sobrejacente, no caso das lesóes em tecidos moles; o neurilemoma é uma lesão altamente radiorresistente, fato que faz com que seja descartado este tipo de terapia. A recorrência é rara e o prognóstico excelente (Pimkhaokham et al. ${ }^{13}$ 2006).

O caso clínico apresentado está em concordância, na maioria de suas características, com os dados clínicos, microscópicos e epidemiológicos encontrados na literatura. $\mathrm{O}$ tratamento adotado, excisão cirúrgica, também se baseou em relatos anteriores de sucesso. $\mathrm{O}$ paciente permanece em proservação, apresentando-se bem, sem sinais de recidiva até o momento, passados 6 meses da intervenção cirúrgica.

\section{CONCLUSÃO}

Como consideraçóes finais, é válido ressaltar que todo nódulo encontrado nos tecidos moles da boca, especialmente na língua, deve ser examinado com atenção e que a análise anátomo-patológica é imprescindível para o diagnóstico final. Para tanto, é fundamental o papel do cirurgiáo-dentista, não só na deteç̧ão, o mais precoce possível, de alteraçóes na boca, mas também como um veículo de divulgação, orientação e incentivo ao autoexame bucal. 


\section{REFERÊNCIAS}

1. Amir R, Altman KW, Zaheer S. Neurilemmoma of the hard palate. J Oral Maxillofac Surg. 2002 Sep; 60(9): 1069-71.

2. Baranovic M, Macan D, Begovic EA, Luksic I, Brajdic D, Manojlovic S. Schwannoma with secondary erosion of mandible: case report with a review of the literature. Dentomaxillofac Radiol. 2006 Nov; 35(6): 456-60.

3. Chi AC, Carey J, Muller S. Intraosseous schwannoma of the mandible: a case report and review of the literature. Oral Surg Oral Med Oral Pathol Oral Radiol Endod. 2003 July: 96(1): 54-65.

4. Feltrin PP, Sugaya NN, Marcucci G, Araujo NS. Neurilemoma da cavidade bucal. Rev Ass Paul Cirurg Dent. 1978 jan./fev.; 32(1): 68-70.

5. Hatziotia JC, Asprides H. Neurilemoma (schwannoma) of the oral cavity. Oral Surg Oral Med Oral Pathol. 1967 Oct; 24(4): 510-26.

6. Kawakami R, Kaneko T, Kodoia M, Matsushita T, Fuginaga Y, Oguchi K, Kurashina K. Schwannoma in the sublingual space. Dentomaxillofac Radiol. 2004 July; 33(4): 259-61.

7. Koizumi Y, Utsunomiya T, Yamamoto H. Cellular schwannoma in the oral mucosa. Acta Otolaryngol. 2002 June; 122(4): 458-62.

8. Krolls SO, McGinnis JP, Quon D. Multinodular versus plexiform neurilemoma of the hard palate - Report of a case. Oral Surg Oral Med Oral Pathol. 1994 Feb; 77(2): 154-7.
9. Martins MD, Taghloubi SA, Bussadori SK, Fernandes KP, Palo RM, Martins MA. Intraosseous schwannoma mimicking a periapical lesion on the adjacent tooth: case report. Int Endod J. 2007 Jan; 40(1): $72-8$

10. Marzola C, Borguetti MJ, Consolaro A. Neurilemmoma if the mandible. J Oral Maxillofac Surg. 1988 Apr; 46(4): 330-4.

11. Nary Filho H, Ribeiro Junior PD, Matsumoto MA, Batista AC, Soares AB, Consolaro A. Neurilemoma no palato duro: relato de caso clínico. BCI. 2002 jan./mar.; 9(33): 37-41.

12. Pfeifle R, Baur DA, Paulino A, Helman J. Schwannoma of the tongue: report of 2 cases. J Oral Maxillofac Surg. 2001 July; 59(7): 802-4.

13. Pimkhaokham A, Piriyasupong P, Swasdison S, Pimkhaokham S, Tung P. Central neurilemmoma of the jaw in concurrence with radicular cyst: a case report. Oral Surg Oral Med Oral Pathol Oral Radiol Endod. 2006 Oct; 102(4): e34-6 Epub 2006 Aug 10.

14. Regezzi JA, Sciubba N. Patologia bucal - correlações clínicopatológicas. $3^{\mathrm{a}}$ ed. Rio de Janeiro: GuanabaraKoogan, 2000.

15. Yamazaki H, Kaneko A, Ota Y, Tsukinoki K. Schwannoma of the mental nerve: usefulness of preoperative imaging: a case report. Oral Surg Oral Med Oral Pathol Oral Radiol Endod. 2004 Jan; 97(1): 122-6.

Recebido em: 17/03/2008

Aceito em24/03/2009 